\title{
Water masers within the G 333.2-0.6 giant molecular cloud
}

\author{
S. L. Breen, ${ }^{1}$ S. P. Ellingsen, ${ }^{1}$ M. Johnston-Hollitt, ${ }^{1}$ \\ S. Wotherspoon, ${ }^{1}$ I. Bains, ${ }^{2,3}$ M. G. Burton, ${ }^{2}$ M. Cunningham ${ }^{2}$ \\ N. Lo, ${ }^{2}$ C. E. Senkbeil ${ }^{1}$ and T. Wong ${ }^{2,4}$ \\ ${ }^{1}$ School of Mathematics and Physics, University of Tasmania, Private Bag 37, Hobart, \\ Tasmania 7001, Australia \\ ${ }^{2}$ School of Physics, University of New South Wales, Sydney, NSW 2052, Australia \\ ${ }^{3}$ Centre for Astrophysics and Supercomputing, Swinburne University of Technology, PO Box \\ 218, Hawthorn, VIC 3122, Australia \\ ${ }^{4}$ Australia telescope National Facility, CSIRO, PO Box 76, Epping, NSW 1710, Australia
}

\begin{abstract}
We report the results of a blind search for $22 \mathrm{GHz}$ water masers in two regions, covering approximately half a square degree, within the G 333.2-0.6 giant molecular cloud. The complete search of the two regions was carried out with the $26 \mathrm{~m}$ Mount Pleasant radio telescope and resulted in the detection of nine water masers, five of which are new detections. Australia Telescope Compact Array (ATCA) observations of these detections have allowed us to obtain positions with arcsecond accuracy, allowing meaningful comparison with infrared and molecular data for the region. We find that for the regions surveyed there are more water masers than either $6.7 \mathrm{GHz}$ methanol, or main-line $\mathrm{OH}$ masers. The water masers are concentrated towards the central axis of the star formation region, in contrast to the $6.7 \mathrm{GHz}$ methanol masers which tend to be located near the periphery. The colours of the GLIMPSE point sources associated with the water masers are slightly less red than those associated with methanol masers. Statistical investigation of the properties of the ${ }^{13} \mathrm{CO}$ and $1.2 \mathrm{~mm}$ dust clumps with and without associated water masers shows that the water masers are associated with the more massive, denser and brighter ${ }^{13} \mathrm{CO}$ and $1.2 \mathrm{~mm}$ dust clumps. We present statistical models that can predict those ${ }^{13} \mathrm{CO}$ and $1.2 \mathrm{~mm}$ dust clumps likely to have associated water masers.
\end{abstract}

Keywords. Masers, ISM: molecules, radio lines: general, stars: formation.

\section{Introduction}

The G333.2-0.6 giant molecular cloud (GMC) is located at a distance of $3.6 \mathrm{kpc}$ (Lockman 1979) and extends approximately 1.2 x 0.6 degrees on the sky. The GMC has been the focus of numerous observations, including $1.2 \mathrm{~mm}$ dust continuum observations by Mookerjea et al. (2004) who identified 95 dust clumps within the region. Observations of ${ }^{13} \mathrm{CO}$ by Bains et al. (2006) identified $61{ }^{13} \mathrm{CO}$ clumps within the GMC and showed that the emission takes the form of a string of knots with the clumps arranged along an axis aligned NW to SE. Complete surveys of the region have been carried out by Ellingsen et al. (1996) for 6.7 GHz methanol masers and Caswell et al. (1980) for 1665 and 1667 $\mathrm{MHz} \mathrm{OH}$ masers. These surveys resulted in the detection of four methanol and three $\mathrm{OH}$ masers within the regions surveyed for water masers. Here we present the results of an untargeted survey within two distinct regions of the G 333.2-0.6 GMC which has resulted in the detection of nine water masers, five of which are new detections. The two regions surveyed cover over half a square degree and encompass much of the high density gas and dust regions of the GMC. For greater detail of the observations and analysis see Breen et al. (2007). 


\section{Discussion and conclusions}

\subsection{Association with other maser species and infrared sources}

We find that within the regions surveyed there are more water than either methanol or $\mathrm{OH}$ masers and that there are relatively few associations between the different maser species. Comparison between the relative locations of the water masers and the $6.7 \mathrm{GHz}$ methanol masers shows that the water masers lie along the main axis of star formation within the GMC while the methanol masers tend to be located near the periphery. Four of the five newly discovered water masers have an associated GLIMPSE point source and the four previously detected water masers are all projected against regions of bright mid-infrared emission. Comparison of the [3.6]-[4.5] colours of the water maser associated GLIMPSE point sources with those associated with methanol masers (Ellingsen 2006) shows that the sources associated with the water masers are clustered toward the less red end of the range observed in the methanol associated sources. This coupled with the relative positions of the water and methanol masers within the GMC lends support to the idea that methanol masers trace an earlier evolutionary phase than water masers.

\section{2. ${ }^{13} \mathrm{CO}$ and $1.2 \mathrm{~mm}$ dust clump analysis}

We have investigated the properties of the ${ }^{13} \mathrm{CO}$ (Bains et al. 2006) and the dust clumps (Mookerjea et al. 2004) that are associated with water maser emission by fitting a Binomial generalised linear model to the maser presence/absence data using the ${ }^{13} \mathrm{CO}$ and dust clump properties as predictors. This analysis has shown that in general water masers are associated with bigger, brighter, more massive and denser ${ }^{13} \mathrm{CO}$ and dust clumps. Stepwise model selection based on the Akaike Information Criteria was used to select the most parsimonious models with the greatest predictive properties for both the ${ }^{13} \mathrm{CO}$ and the dust clumps. The estimated regression relations for the ${ }^{13} \mathrm{CO}$ and dust clumps are

$$
\log \frac{p_{i}}{1-p_{i}}=-21.2018+1.3037 x_{\text {integrated }}+8.0589 x_{\text {radius }}-1.2290 x_{\text {mass }},
$$

and

$$
\log \frac{p_{i}}{1-p_{i}}=-11.477+9.174 x_{\text {radius }},
$$

respectively, where $p_{i}$ is the probability of maser presence in the $i^{\text {th }}$ clump and $x_{\text {integrated }}$, $x_{\text {radius }}$ and $x_{\text {mass }}$ represent clump properties integrated flux density of the clump peak $\left(10 \mathrm{~K} \mathrm{~km} \mathrm{~s}^{-1}\right)$, mass $\left(10^{3} \mathrm{M}_{\odot}\right)$ and radius (pc). These models have low misclassification rates and allow the probability of maser presence within a given clump to be calculated, enabling efficient targeted searches to be carried out where suitable data is available.

\section{References}

Bains, I., Wong, T., Cunningham, M., Sparks, P., Brisbin, D., Calisse, P., Dempsey, J. T., Deragopian, G., Ellingsen, S., Fulton, B., Herpin, B., Jones, P., Kouba, Y., Kramer, C., Ladd, E. F., Longmore, S. N., McEvoy, J., Maller, M., Minier, V., Mookerjea, B., Phillips, C., Purcell, C. R., Walsh, A., Voronkov, M. A., Burton, M. G. 2006, MNRAS 367, 1609

Breen, S. L., Ellingsen, S. P, Johnston-Hollitt, M., Wotherspoon, S., Bains, I., Burton, M. G., Cunningham, M., Lo, N., Senkbeil, C. E., Wong, T. 2007 MNRAS in press

Caswell, J. L., Haynes, R. F., Goss, W.M 1980, Aust. J. Phys., 33, 639

Ellingsen, S. P., von Bibra, M. L., McCulloch, P. M., Norris, R. P., Deshpande, A. A., Phillips, C. J. $1996, M N R A S 280,378$

Ellingsen, S. P. 2006, ApJ 638, 241

Lockman, F. J. 1979, ApJ 232, 761

Mookerjea, B., Kramer, C., Nielbock, M., Nyman, L. 2004, A $\mathscr{E}$ A 426, 119 